
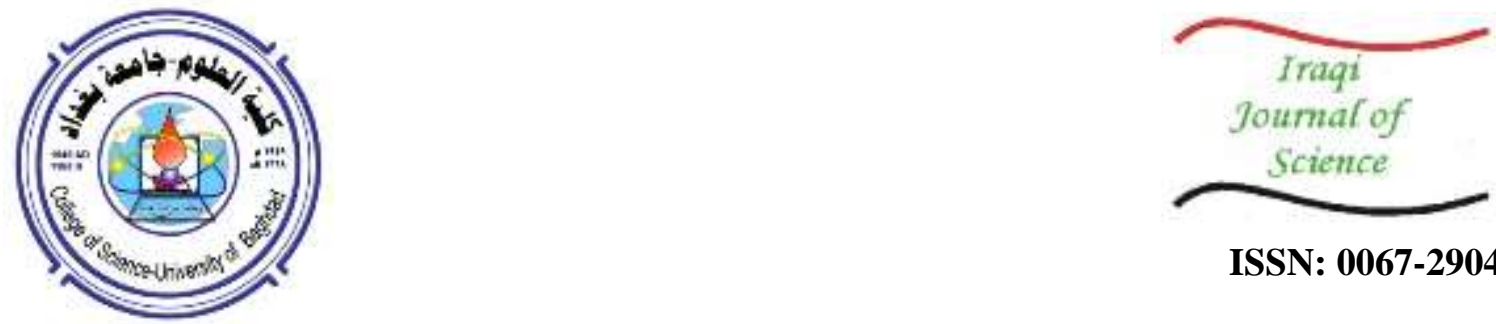

ISSN: 0067-2904

\title{
Maximal Ideal Graph of Commutative Rings
}

\author{
F. H. Abdulqadr \\ Department of Mathematics, College of Education, University of Salahaddin, Erbil, Iraq
}

Received: 18/9/2019

Accepted: 19/11/2019

\begin{abstract}
In this paper, we introduce and study the notion of the maximal ideal graph of a commutative ring with identity. Let $\mathrm{R}$ be a commutative ring with identity. The maximal ideal graph of $\mathrm{R}$, denoted by $\mathrm{MG}(\mathrm{R})$, is the undirected graph with vertex set, the set of non-trivial ideals of $R$, where two vertices $I_{1}$ and $I_{2}$ are adjacent if $I_{1} \neq I_{2}$ and $I_{1}+I_{2}$ is a maximal ideal of $R$. We explore some of the properties and characterizations of the graph.
\end{abstract}

Keywords: The maximal ideal graph of a commutative ring R, maximal ideals and connected graphs.

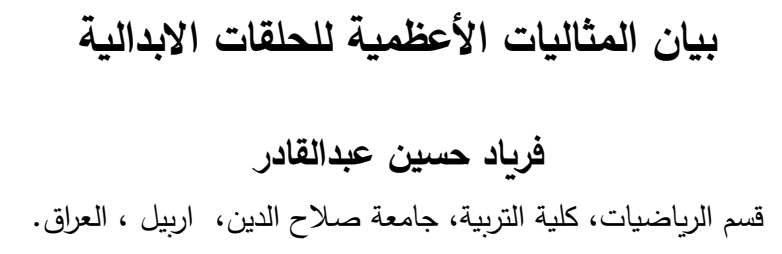

الخلاصة

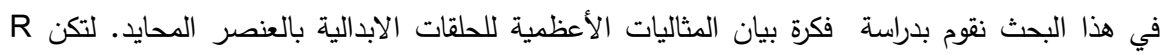

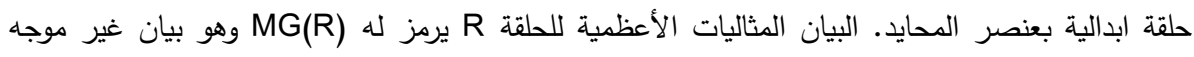

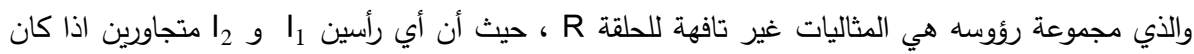

ال

\section{Introduction}

البيان.

The graphs assigned to a commutative ring have been studied by many mathematicians. The zero divisor graph of commutative rings was first introduced by Beck in [1]. After that, many mathematicians studied such graphs [2- 5].

Throughout this paper, $\mathrm{R}$ will be a commutative ring with identity. We introduce and investigate the notion of maximal ideal graph of a commutative ring $R$ with identity, which is denoted by MG(R). It is the undirected graph with vertex set, the set of non-trivial ideals of $R$, where two vertices $I_{1}$ and $I_{2}$ are adjacent if $I_{1} \neq I_{2}$ and $I_{1}+I_{2}$ are maximal ideals of R. First, we explore some of the properties and characterizations of these graphs. For instance, the rings $R$, for which the graph $M G(R)$ is star or complete bipartite, are characterized. Next, we study the planarity as well as the connectivity of MG(R). It is shown that MG(R) is a connected graph and diam (AG) $(R) \leq 3$.

We recall some definitions in graph theory which are needed in our work $[6,7]$.

The neighborhood of a vertex $\mathrm{v}$ in the graph $\mathrm{G}$, denoted by $\mathrm{N}(\mathrm{v})$, is the set of vertices adjacent to $\mathrm{v}$. The degree of a vertex $v$ of the graph $G$, denoted by $\operatorname{deg}_{G}(v)$, is the number of edges incident to $v$. A

*Email: fryad.abdulqadr@su.edu.krd 
graph $\mathrm{G}$ is a complete graph if every two of its vertices are adjacent. A complete graph of order $\mathrm{n}$ is denoted by $K_{n}$. A graph $G$ is $n$ - partite, $n \geq 1$, if it is possible to partition $V(G)$ into $n$ subsets $V_{1}, V_{2}$, $\ldots, V_{n}$ ( called partite sets) such that every element of $E(G)$ joins a vertex of $V_{i}$ to a vertex of $V_{j}, i \neq j$. A complete bipartite graph with exactly two partitions of size $m$ and $n$ is denoted by $K_{m, n}$. A graph $G$ is said to be star if $G=K_{1, n}$. Two vertices $u$ and $v$ of a graph $G$ are said to be connected in $G$ if there exists a path between them. A graph $\mathrm{G}$ is called connected if all pairs of its vertices are connected. Let $\mathrm{G}$ be a connected graph. The distance between a pair of vertices $\mathrm{u}$ and $\mathrm{v}$ of $\mathrm{G}$, denoted by $\mathrm{d}(\mathrm{u}, \mathrm{v})$, is the length of the shortest u-v path in G. The diameter, eccentricity, and radius of a connected graph $\mathrm{G}$ are defined by $\operatorname{diamG}=\operatorname{Max}\{\mathrm{d}(\mathrm{u}, \mathrm{v}): \mathrm{u}, \mathrm{v} \in \mathrm{V}(\mathrm{G})\}, \quad \mathrm{e}(\mathrm{v})=\operatorname{Max}\{\mathrm{d}(\mathrm{u}, \mathrm{v})$ : for all $\mathrm{u} \in \mathrm{V}(\mathrm{G})\}$ and $\operatorname{rad}(\mathrm{G})=\operatorname{Min}\{\mathrm{e}(\mathrm{v}): \mathrm{v} \in \mathrm{V}(\mathrm{G})\}$, respectively. A vertex $\mathrm{v}$ of a connected graph $\mathrm{G}$ is a cut-vertex if the components of G-v are more than the components of G. The girth of a graph $\mathrm{G}$ is the length of the shortest cycle in $\mathrm{G}$. A k-coloring of a graph $\mathrm{G}$ is a function $\mathrm{C}: \mathrm{V}(\mathrm{G}) \rightarrow\{1,2, \ldots, \mathrm{k}\}$ such that $\mathrm{C}(\mathrm{u}) \neq \mathrm{C}(\mathrm{v})$ whenever $\mathrm{u}$ is adjacent to $\mathrm{v}$. If a $\mathrm{k}$-coloring of $\mathrm{G}$ exists, then $\mathrm{G}$ is k-colorable. The chromatic number of $\mathrm{G}$ is defined by $\chi(\mathrm{G})=\min \{\mathrm{k} ; \mathrm{G}$ is k-colorable $\}$. A complete sub-graph $\mathrm{K}_{\mathrm{n}}$ of a graph $\mathrm{G}$ is called a clique, and $\omega(\mathrm{G})$ is the clique number of $\mathrm{G}$, which is the greatest integer $r \geq 1$ such that $\mathrm{K}_{\mathrm{r}} \subseteq \mathrm{G}$. $A$ graph $\mathrm{G}$ is called a planar graph if it can be drawn on a plane in such a way that any two of its edges either meet only at their end vertices or do not meet at all. A graph $G$ is perfect if every induced subgraph $\mathrm{H}$ of $\mathrm{G}$ satisfies $\chi(\mathrm{H})=\omega(\mathrm{H})$. A graph is a split graph if it can be partition in an independent set and a clique.

Throughout this work, we use $\mathrm{J}_{R}, \mathrm{I}_{\mathrm{R}}, \mathrm{m}_{\mathrm{R}}$ and $\mathrm{M}_{\mathrm{R}}$ to denote the Jacobson radical, the set of nontrivial ideals, the set of minimal and maximal ideals of a ring $\mathrm{R}$, respectively.

\section{The Maximal Ideal Graph of $\mathbf{R}$}

In this section, we introduce the notion of the maximal ideal graph of a commutative ring with identity. We illustrate this concept by examples and remarks and give some of its properties and characterizations.

Definition2.1: Let $\mathrm{R}$ be a commutative ring with identity. The maximal ideal graph of $\mathrm{R}$, denoted by $\mathrm{MG}(\mathrm{R})$, is the undirected graph with vertex set, the set of all non-trivial ideals of $\mathrm{R}$, where two vertices $I_{1}$ and $I_{2}$ are adjacent if $I_{1} \neq I_{2}$ and $I_{1}+I_{2}$ are maximal ideals of $R$.

We begin with the following easy result which may be needed in the sequel.

\section{Lemma2.2:}

1. Every non-maximal ideal is adjacent to at least one maximal ideal in $\mathrm{MG}(\mathrm{R})$.

2. If $P_{1}, P_{2}, \ldots, P_{n} \in M_{R}$ such that $\bigcap_{i=1}^{n} P_{i} \notin M_{R} \cup\{(0)\}$, then the ideal $\bigcap_{i=1}^{n} P_{i}$ is adjacent to every $\mathrm{P} \in \mathrm{M}_{\mathrm{R}}$ in $\mathrm{MG}(\mathrm{R})$.

\section{Proof:}

1. Let $J \in V(M G(R)) \backslash M_{R}$. Then $J \subset Q$, for some $Q \in M_{R}$. Obviously, $I+Q=Q$. Thus $I$ is adjacent to $Q$.

2. The proof follows from the first part of the Lemma2.2.

Example1: Consider the ring $\mathrm{z}_{60}$. The graph $\mathrm{MG}\left(\mathrm{z}_{60}\right)$ is:

(2)

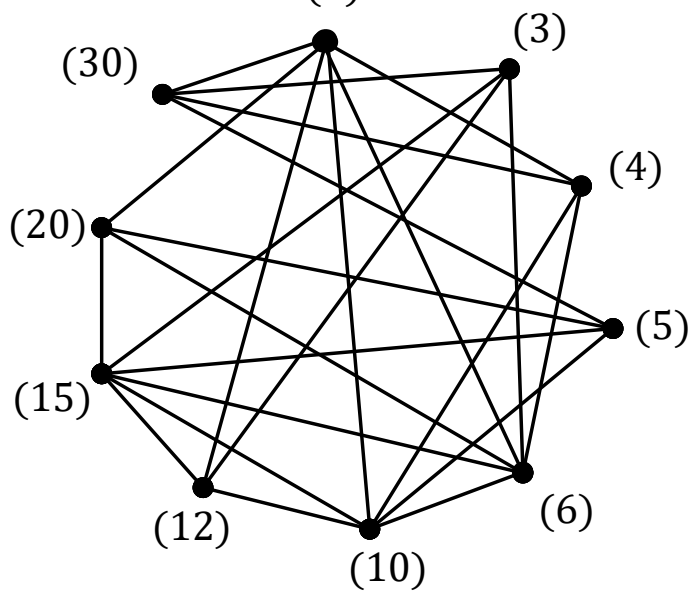

Figure 1-The graph $\mathrm{MG}\left(\mathrm{z}_{60}\right)$ 
It is obvious from Figure-1 that every non- maximal ideal is adjacent to a maximal ideal.

Remark2.3: The co-maximal ideals of $R$ are not adjacent in $M G(R)$.

The next main result shows the adjacency between ideal vertices of $M G(R)$.

Theorem 2.4: Let I, J and $P$ be three distinct vertices of $M G(R)$ with $P \in M_{R}$. Then:

1. $P \in N(I) \cap N(J) \Leftrightarrow P \in N(I+J)$, where $I+J \neq P, R$.

2. IC $\mathrm{J}_{\mathrm{R}} \Rightarrow \mathrm{P} \in \mathrm{N}(\mathrm{I})$

3. $\mathrm{I} \subset \mathrm{J} \wedge \mathrm{J} \notin \mathrm{M} \Rightarrow \mathrm{I} \notin \mathrm{N}(\mathrm{J})$

4. $I \in N(J L) \Rightarrow I \in N(J \cap L) \cap N(J)$, for every vertices $L$ in which $L J \neq(0)$.

Proof:

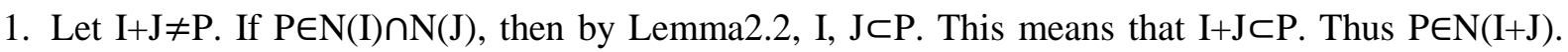
Similarly, $\mathrm{P} \in \mathrm{N}(\mathrm{I}+\mathrm{J})$ leads to $\mathrm{P} \in \mathrm{N}(\mathrm{I}) \cap \mathrm{N}(\mathrm{J})$.

2. Let $\mathrm{I} \subset \mathrm{J}_{\mathrm{R}}$. Then I $\subset \mathrm{I}+\mathrm{J}_{\mathrm{R}}=\mathrm{J}_{\mathrm{R}} \subseteq \mathrm{P}$. By Lemma2.2, $\mathrm{P} \in \mathrm{N}(\mathrm{I})$.

Similarly, we can show the other parts of Theorem2.4.

Proposition2.5: If $\{I, J\} \in E(M G(R))$ with $I, J \notin M_{R}$, then there exists a unique $M \in M_{R}$ such that $\mathrm{M} \in \mathrm{N}(\mathrm{I}) \cap \mathrm{N}(\mathrm{J})$.

Proof: Suppose that $M_{1}, M_{2} \in M_{R}$ and each of $I$ and $J$ are adjacent to both $M_{1}$ and $M_{2}$ in $M G(R)$. Then by Lemma2.2, $I, J \subset M_{1} \cap M_{2}$. Since $I+J \in M_{R}$, then $M_{1}=I+J=M_{2}$.

The next result shows that the degree of maximal ideals determines the finiteness of MG(R).

Proposition 2.6: Let $R$ be Artinian. If $\operatorname{deg} I<\infty$, for every $I \in M_{R}$, then $M G(R)$ is a finite graph.

Proof: Since $R$ is Artinian ([8], Theorem8.7), then $R$ is isomorphic to $R_{1} \times R_{2} \times \cdots \times R_{n}$, where $\left(R_{i}, P_{i}\right)$ is a local Artinian ring. The maximally of $I$ gives that $I=R_{1} \times R_{2} \times \cdots \times R_{i-1} \times P_{i} \times R_{i+1} \times \cdots \times R_{n}$, where $1 \leq \mathrm{i} \leq \mathrm{n}$. Since $\operatorname{degI}$ is finite, then $\mathrm{I}_{\mathrm{R}_{\mathrm{i}}}$ is finite. Thus $\mathrm{MG}(\mathrm{R})$ is a finite graph.

The next result gives the conditions on $\mathrm{MG}(\mathrm{R})$ for which $\mathrm{R}$ is a local ring.

Poposition2.7: If $M G(R) \cong K_{n}$ or $M G(R) \cong K(n, 1)$, where $n \in Z^{+}$, then $R$ is local.

Proof: If $M G(R) \cong K_{n}$, then by Remark2.4, $R$ is local. Let $M G(R)$ be a star with center I. If $M G(R)$ consists of only one edge, then it refers to completeness case. Assume that $|M G(R)| \geq 3$. If $I \notin M_{R}$, then by Lemma2.2, $\mathrm{V}(\mathrm{MG}(\mathrm{R})) \backslash\{\mathrm{I}\}=\mathrm{M}_{\mathrm{R}}$. Thus $\mathrm{I}=\mathrm{J}_{R} \neq(0)$. Now, suppose that $\mathrm{P}, \mathrm{S} \in \mathrm{M}_{\mathrm{R}}$ with $\mathrm{P} \neq \mathrm{S}$. Obviously, $(0) \neq P S \notin M_{R}$. Thus $P S=I=J_{R}$. This contradicts that $|M G(R)| \geq 3$. Therefore, $I \in M_{R}$. Again by Lemma2.2, $\mathrm{M}_{\mathrm{R}}=\{\mathrm{I}\}$. Thus the proof is completed.

The converse of Proposition2.7 will be true if $\mathrm{V}(\mathrm{MG}(\mathrm{R}))$ is a totally ordered set. We illustrate it in the following result.

Proposition 2.8: If $V(M G(R))$ is a totally ordered set, then $M G(R)$ is a star.

Proof: Since $\mathrm{V}(\mathrm{MG}(\mathrm{R}))$ is a totally ordered set, then $\mathrm{MG}(\mathrm{R})$ contains a vertex I which is adjacent to each other vertex. If $J \neq I$ and $P \neq I$ are two distinct vertices of $M G(R)$, then either $P \subset J$ or $J \subset P$. For both cases, $J$ and $K$ are not adjacent vertices. Thus $M G(R)$ is a star with center I.

Corollary2.9: For any prime number $\mathrm{p}$, the graph $\mathrm{MG}\left(\mathrm{z}_{\mathrm{p}} \mathrm{n}\right)$ is star.

Proof: It follows from Proposition2.8.

Now, we give the condition for which $\mathrm{MG}(\mathrm{R})$ be a complete bipartite, as follows.

Theorem2.10: Let $J_{R} \notin\{(0)\} \cup M_{R}$. Then $M G(R) \cong K_{m, n} ; m, n \in Z^{+}$if and only if $I_{R}-M_{R} \subseteq J_{R}$.

Proof: Let $I_{R}-M_{R} \subseteq J_{R}$. Choose $V_{1}=M_{R}$ and $V_{2}=\left\{I \in V(M G(R))\right.$ : $\left.I \subseteq J_{R}\right\}$. From Lemma2.2, every two vertices in $V_{1}$ are independent with respect to the graph $M G(R)$. Since $|M G(R)| \neq 1$, then $J_{R} \notin M_{R}$. Thus $I+J \notin M_{R}$ for every $I, J \in V_{2}$. This means that every two vertices in $V_{2}$ are independent with respect to the graph $\mathrm{MG}(\mathrm{R})$. On the other hand, Theorem2.4 mentions that every $I \in V_{1}$ is adjacent to each $\mathrm{J} \in \mathrm{V}_{2}$. This ends the proof.

Conversely, if $M G(R)$ is a complete bipartite with partite sets $W_{1}$ and $W_{2}$, we can prove that $W_{i}=M_{R}$ and $\mathrm{W}_{\mathrm{j}}=\left\{\mathrm{I} \in \mathrm{V}(\mathrm{MG}(\mathrm{R})): \mathrm{I} \subseteq \mathrm{J}_{\mathrm{R}}\right\}$, for $\mathrm{i}, \mathrm{j}=1,2$ with $\mathrm{i} \neq \mathrm{j}$. This completes the proof.

Corollary2.11: Let $J_{R} \notin M_{R} \cup\{(0)\}$. If $M G(R)$ is not a complete bipartite, then $M G(R)$ is a 3-partite graph.

Proof: Since $M G(R)$ is not a complete bipartite, then by Theorem2.10, $I \nsubseteq J_{R}$, for some $I \in M G(R) \backslash M_{R}$. We set $V_{1}=M_{R}, V_{2}==\left\{I \in V(M G(R)): I \subseteq J_{R}\right\}$ and $V_{3}=V(M G(R))\left(\left(V_{1} \cup V_{2}\right)\right.$. It is not difficult to show that every two vertices in $V_{i}$ are independent, for $i=1,2,3$. Thus $M G(R)$ is a 3-partite graph.

Example2: The graph $\mathrm{MG}\left(\mathrm{z}_{36}\right)$ is a 3-partite graph, as the following figure shows: 
(3)

(2)

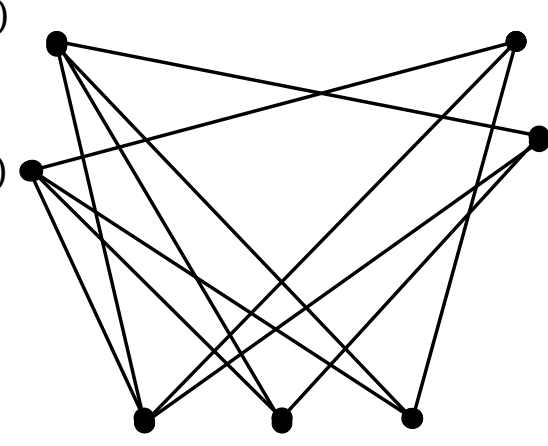

(6) (12)
(4)

(18)

Figure 2- The graph $\mathrm{MG}\left(\mathrm{z}_{36}\right)$

Now we are at the position of the following main result.

Theorem2.12: $\operatorname{Letm}_{R} \neq \emptyset$. If $\mathrm{V}(\mathrm{MG}(\mathrm{R}))=\mathrm{m}_{\mathrm{R}} \cup \mathrm{M}_{R}$, then:

1. The graph $\mathrm{MG}(\mathrm{R})$ is split.

2. The graph $M G(R)$ is perfect.

3. The clique number of $M G(R)$ is $\omega(M G(R))=\max \left\{\left|m_{R}\right|,\left|m_{R}\right|+1\right\}$.

\section{Proof:}

1. Let $A$ be the induced subgraph of $M G(R)$ bym $_{R}$. Let $S, T \in m_{R}$ with $S \neq T$. Obviously, $S+T \neq R$. If we assume that $S+T \in m_{R}$, then $S=S+T=T$, which is a contradiction. Therefore, $S+T \in M G(R)$. Thus A is a complete graph. From Remark2.3, the vertices in $M_{R}$ are independent. Hence MG(R) is a split graph.

2. Let $C: I_{1}, I_{2}, \cdots I_{2 n+1}, I_{1}$ be an induced cycle in $M G(R)$ with $n \geq 2$. If $C$ does not contain any maximal ideal vertex, then by the first part of Theorem2.12, $\left\{\mathrm{I}_{1}, \mathrm{I}_{3}\right\} \in \mathrm{E}(\mathrm{MG}(\mathrm{R}))$, which is a contradiction. Let $I_{1} \in M_{R}$. Obviously, $I_{2 n+1}, I_{2} \notin M_{R}$. Then they are adjacent in $M G(R)$, which is a contradiction. Now, assume that $C^{\prime}$ is an induced odd cycle in $\overline{M G(R)}$ of length $n \geq 5$. Then $C^{\prime}$ contains at least $P, Q \in M_{R}$ with $\mathrm{P} \neq \mathrm{Q}$ such that they are not adjacent in $\mathrm{C}^{\prime}$. From Lemma2.2, $\mathrm{P}$ and $\mathrm{Q}$ are adjacent in $\overline{\mathrm{MG}(\mathrm{R})}$. This contradics Lemma2.2. Hence, by the strong perfect graph theorem in [9], MG(R) is a perfect graph.

3. The proof follows from the first part of Theorem2.12.[10]

Example3: Consider the ring $\mathrm{z}_{12}$. The following graph shows that $\mathrm{MG}\left(\mathrm{z}_{12}\right)$ is a split and perfect graph. Also $\omega\left(\mathrm{MG}\left(\mathrm{z}_{12}\right)\right)=\left|\mathrm{m}_{\mathrm{R}}\right|+1=3$.

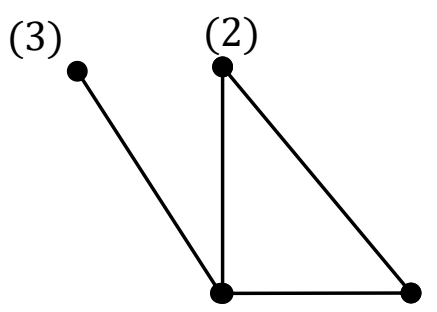

(6)

Figure 3- The graph $\mathrm{MG}\left(\mathrm{z}_{12}\right)$

In the next result, we find the girth of $\mathrm{MG}(\mathrm{R})$.

Theorem 2.13: Let $J_{R} \neq(0)$. The girth $g(M G(R))$ is either 3,4 , or $\infty$.

Proof: If $M G(R)$ contains an edge $\{S, T\}$ with $S, T \notin M_{R}$, then $S, T \neq S+T \in M_{R}$. Thus $S+T$ is adjacent to both $S$ and T. This means that $C: S, T, S+T, S$ is a cycle in $M G(R)$. In this case, $g(M G(R))=3$. Suppose that for every $\{I, J\} \in E(M G(R))$, either $I \in M_{R}$ or $J \in M_{R}$. If $M G(R)$ does not possess any cycle, then $g(M G(R))=\infty$. Now, suppose that $C_{n}: I_{1}, I_{2}, \ldots, I_{n}, I_{1}$ is a cycle in $M G(R)$ of length $n$. Since the maximal ideals are not adjacent in $\mathrm{MG}(\mathrm{R})$, the vertices of $\mathrm{C}$ are alternatively maximal and nonmaximal ideals. Consequently, $\mathrm{J}_{\mathrm{R}} \notin \mathrm{M}_{\mathrm{R}}$. Let $\mathrm{I}_{1} \in \mathrm{M}_{\mathrm{R}}$. From Lemma2.2, $\mathrm{J}_{\mathrm{R}}$ is adjacent to each of $\mathrm{I}_{1}, \mathrm{I}_{3}$ 
and $I_{5}$. If $I_{2}=J_{R}$, then $C^{\prime}: I_{2}, I_{3}, I_{4}, I_{5}, I_{2}$ is a cycle in $M G(R)$. If $J_{R} \neq I_{2}$, then $C^{\prime \prime}: J_{R}, I_{1}, I_{2}, I_{3}, J_{R}$ is a cycle in $M G(R)$. From both cases, we have shown that $g(M G(R))$ is either 3 or 4 .

The next result shows the upper bound of clique number of $M G(R)$.

Proposition2.14: The clique of $M G(R)$ contains in an its induced subgraph by $\{I \in V(M G(R))$ : $I \subseteq P\}$, for exactly one $\mathrm{P} \in \mathrm{M}_{\mathrm{R}}$.

Proof: Let $\mathrm{G}$ be the clique of $\mathrm{MG}(\mathrm{R})$. Since the co-maximal ideals are not adjacent in $\mathrm{MG}(\mathrm{R}), \mathrm{G}$ has at most one maximal ideal. The adjacency of every two vertices of $G$ and Proposition2.5 illustrates that there exists exactly one $P \in M_{R}$ for which $G$ is a subgraph of the graph induced by $\{I \in V(M G(R))$ : $\mathrm{I} \subseteq \mathrm{P}\}$.

\section{The Planarity of MG(R)}

First, we find the clique number of MG(R).

Proposition3.1: If the subgraph induced by $\{I \in V(M G(R))$ : $I \subseteq P\}$ is planar, for every $P \in M_{R}$, then $\omega(\mathrm{MG}(\mathrm{R}))$ is either 2 or 3 or 4 .

Proof: The proof follows from Proposition2.14 and Koratowsky theorem [6].

In the next result, we show that $\mathrm{MG}(\mathrm{R})$ is a planar graph under a certain condition on vertex set of MG(R).

Theorem3.2: If $\mathrm{V}(\mathrm{MG}(\mathrm{R}))=\mathrm{m}_{R} \cup \mathrm{M}_{R}$ is finite and $\left|m_{R}\right| \leq 3$, then the graph $M G(R)$ is planar.

Proof: To show that $M G(R)$ is planar, we refer to Koratowsky theorem. Since $\left|m_{R}\right| \leq 3$, then any subgraph of $M G(R)$ induced by five vertices is not complete. This means that $M G(R)$ does not contain any complete subgraph $K_{5}$. If we assume that $M G(R)$ contains a $K_{3.3}$ with partite sets $V_{1}=\left\{I_{1}, I_{2}, I_{3}\right\}$ and $\mathrm{V}_{2}=\left\{\mathrm{J}_{1}, \mathrm{~J}_{2}, \mathrm{~J}_{3}\right\}$, then by Lemma2.2 either $\mathrm{V}_{1} \subseteq \mathrm{M}_{\mathrm{R}}$ or $\mathrm{V}_{2} \subseteq \mathrm{M}_{\mathrm{R}}$. Assume that $\mathrm{V}_{1} \subseteq \mathrm{M}_{\mathrm{R}}$. Then $\mathrm{V}_{2} \subseteq \mathrm{m}_{\mathrm{R}}$. From Proposition2.5, any two of $\mathrm{L}, \mathrm{M}$ and $\mathrm{N}$ are independent. This contradicts that every minimal ideal are adjacent in $\mathrm{MG}(\mathrm{R})$. Therefore, $\mathrm{MG}(\mathrm{R})$ is a planar graph.

The next result demonstrates that the planarity of $M G(R)$ limits the order of $M_{R}$.

Proposition3.3: Let $J_{R} \neq(0)$. If $M G(R)$ is planar graph, then $\left|M_{R}\right| \leq 4$.

Proof: Let $M G(R)$ be a planar graph. Assume by contrary that $M G(R)$ has at least five distinct maximal ideals, say M, N, P, Q and S. Obviously, any one of the vertices MNP, MNPQ and MNPQS are non-zero ideals and adjacent to each of ideals $M, N$ and $P$ in $M G(R)$. Thus $M G(R)$ contains a complete bipartite graph $K_{3,3}$. This contradicts the Koratowsky theorem. Therefore $\left|M_{R}\right| \leq 4$.

Before closing this section, we give the following main result.

Theorem3.4: Let $R \cong R_{1} \times R_{2} \times \cdots \times R_{n}$, with $R_{1}, R_{2}, \cdots, R_{n}$ are distinct fields. Then $M G(R)$ is planar graph if and only if $n \leq 4$.

Proof: Let $M G(R)$ be a planar graph. Assume that $n>4$. Obviously, $(0) \times R_{2} \times \cdots \times R_{n} \in M_{R}$ and the sum of every two of ideals $(0) \times \mathrm{R}_{2} \times \cdots \times \mathrm{R}_{\mathrm{n}},(0) \times(0) \times \mathrm{R}_{3} \times \cdots \times \mathrm{R}_{\mathrm{n}},(0) \times \mathrm{R}_{2} \times(0) \times \mathrm{R}_{4} \times \cdots \times \mathrm{R}_{\mathrm{n}}$, $(0) \times R_{2} \times R_{3} \times(0) \times R_{5} \times \cdots \times R_{n},(0) \times R_{2} \times R_{3} \times R_{4} \times(0) \times \cdots \times R_{n}$ is equal to $(0) \times R_{2} \times \cdots \times R_{n}$. Then $M G(R)$ contains a complete sub-graph of order 5 . This contradicts the planarity of $M G(R)$. Therefore, $\mathrm{n} \leq 4$.

Conversely, let $n \leq 4$. Clearly, $E(M G(R))=\varnothing$, when $n \in\{1,2\}$. Now, suppose that $n=3$. Then $V(M G(R))$ consists of $\mathrm{I}_{1}=\mathrm{R}_{1} \times(0) \times(0), \mathrm{I}_{2}=(0) \times \mathrm{R}_{2} \times(0), \mathrm{I}_{3}=(0) \times(0) \times \mathrm{R}_{3} \mathrm{I}_{4}=\mathrm{R}_{1} \times \mathrm{R}_{2} \times(0), \mathrm{I}_{5}=\mathrm{R}_{1} \times(0) \times \mathrm{R}_{3}$ and $I_{6}=(0) \times R_{2} \times R_{3}$, and the graph $M G(R)$ is:

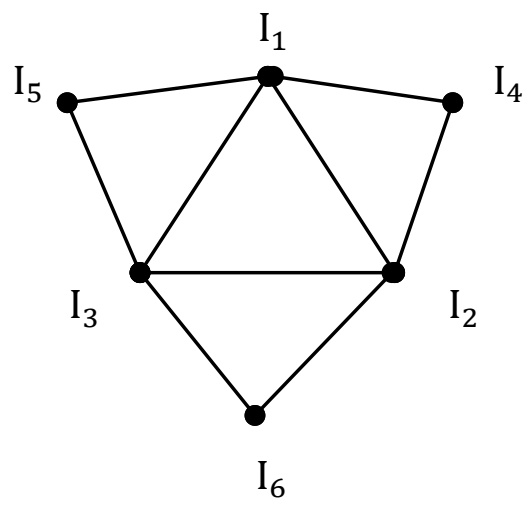

Figure 4- The graph $M G\left(R_{1} \times R_{2} \times R_{3}\right)$ 
Obviously, $\mathrm{MG}(\mathrm{R})$ is planar graph, when $\mathrm{n}=3$.

Now, suppose that $n=4$. The maximal ideal vertices of $M G(R)$ are $(0) \times R_{2} \times R_{3} \times R 4, R 1 \times$ (0) $\times R_{3} \times R_{4}, R_{1} \times R_{2} \times(0) \times R_{4}$ and $R_{1} \times R_{2} \times R_{3} \times(0)$, and the other vertices are $(0) \times(0) \times R_{3} \times R_{4}$, $(0) \times \mathrm{R}_{2} \times(0) \times \mathrm{R}_{4},(0) \times \mathrm{R}_{2} \times \mathrm{R}_{3} \times(0), \mathrm{R}_{1} \times(0) \times(0) \times \mathrm{R}_{4}, \mathrm{R}_{1} \times \mathrm{R}_{2} \times(0) \times(0), \mathrm{R}_{1} \times(0) \times \mathrm{R}_{3} \times(0)$, $\mathrm{R}_{1} \times(0) \times(0) \times(0),(0) \times \mathrm{R}_{2} \times(0) \times(0),(0) \times(0) \times \mathrm{R}_{3} \times(0),(0) \times(0) \times(0) \times \mathrm{R}_{4}$. This graph does not contain $\mathrm{K}_{5}$. Also, for every three distinct vertices $\mathrm{I}$, $\mathrm{J}$ and $\mathrm{K}$ of $\mathrm{MG}(\mathrm{R})$, there exists at most two vertices adjacent to each of $I, J$ and $K$. Thus $M G(R)$ does not contain $K(3,3)$. In this case, $M G(R)$ is a planar graph.

\section{The Connectivity of MG(R)}

We start this section with the following result.

Theorem4.1: Let $\mathrm{R}$ be a finite non-local ring with $\mathrm{MG}(\mathrm{R})$ ) is a non-empty graph. Then every two vertices are disconnected if and only if $R=R_{1} \times R_{2}$, where $R_{1}$ and $R_{2}$ are fields.

Proof: If $R=R_{1} \times R_{2}$ with $R_{1}$ and $R_{2}$ are fields, then $V(M G(R))=\left\{(0) \times R_{2}, R_{1} \times(0)\right\}$. Obviously, $(0) \times R_{2}$ and $\mathrm{R}_{1} \times(0)$ are not adjacent in $\mathrm{MG}(\mathrm{R})$.

Conversely, suppose that every two vertices are disconnected. Since $\mathrm{R}$ is a finite non-local ring, then $R \cong R_{1} \times R_{2} \times \cdots \times R_{n}$, where $\left(R_{i}, P_{i}\right)$ is a local ring for every $i=1,2, \ldots, n$ and $n \geq 2$. If $P_{1} \neq(0)$, then $\left(P_{1} \times R_{2} \times \cdots \times R_{n}\right)+\left(P_{1} \times P_{2} \times \cdots \times R_{n}\right) \in M_{R}$, which is a contradiction. Hence $P_{1}=(0)$. Similarly, $\mathrm{P}_{2}=\mathrm{P}_{3}=\cdots=\mathrm{P}_{\mathrm{n}}=(0)$. Thus $\mathrm{R}_{1}, \mathrm{R}_{2}, \ldots, \mathrm{R}_{\mathrm{n}}$ are fields. If $\mathrm{n} \geq 3$, then $(0) \times \mathrm{R}_{2} \times \cdots \times \mathrm{R}_{\mathrm{n}}$ and $(0) \times(0) \times \mathrm{R}_{3} \times \cdots \times \mathrm{R}_{\mathrm{n}}$ are adjacent in $\mathrm{MG}(\mathrm{R})$, which is a contradiction. Therefore, $\mathrm{n}=2$.

In the next main result, we investigate the connectivity of $\mathrm{MG}(\mathrm{R})$.

Theorem4.2: If every two distinct maximal ideals of $R$ have a non-zero intersection, then $M G(R)$ is connected with diamMG(R) $\leq 3$.

Proof: Let $K, L \in V(M G(R))$ with $K \neq L$. If $\{K, L\} \in E(M G(R))$, then they are connected. Suppose that $\{K, L\} \notin E(M G(R))$. Then either $K+L=R$ or $K+L \subset P$, for some $P \in M_{R}$. If $K+L \subset P$, then by Lemma2.2, $\mathrm{P}_{2}: \mathrm{K}, \mathrm{P}, \mathrm{L}$ is a path in $\mathrm{MG}(\mathrm{R})$. If $\mathrm{K}+\mathrm{L}=\mathrm{R}$, then at least one of $\mathrm{K}$ and $\mathrm{L}$ is a maximal ideal and neither $K \subset L$ nor $L \subset K$. Assume that $K \in M_{R}$. If $L \in M_{R}$, again by Lemma2.2, $P_{2}^{\prime}: K, K \cap L, L$ is a path in $M G(R)$. Let $L \notin M_{R}$. Then there exists $M \in M_{R}$ such that $L$ is adjacent to $P$. If $P=K$, then $K$ is adjacent to $L$. Let $K \neq P$. Then $P_{3}: K, K \cap P, P, L$ is a path in $M G(R)$. From each case, we have shown that $K$ and $\mathrm{L}$ are connected and $\mathrm{d}(\mathrm{K}, \mathrm{L}) \leq 3$. Thus $\mathrm{MG}(\mathrm{R})$ is connected with diamMG(R) $\leq 3$.

Observe that the graph MG(R) may not be connected, when two distinct maximal ideals of $\mathrm{R}$ have a zero intersection.

Example4: Consider the ring $\mathrm{z}_{6}$. Obviously, the following graph is disconnected.

Figure 5- The graph MG $\left(\mathbf{z}_{6}\right)$

Next, we turn to the following result.

Proposition4.3: If $\mathrm{R}$ is a principal ideal ring in which every two distinct maximal ideals of $\mathrm{R}$ have a non-zero intersection, then $\operatorname{diamMG}(\mathrm{R}) \leq 2$.

Proof: From Theorem4.2, d $(\mathrm{P}, \mathrm{Q}) \leq 2$, for every $\mathrm{P}, \mathrm{Q} \in \mathrm{V}(\mathrm{MG}(\mathrm{R}))$ with $\mathrm{P} \neq \mathrm{Q}$, except for the possibility that $\mathrm{P}+\mathrm{Q}=\mathrm{R}$ and $\{\mathrm{P}, \mathrm{Q}\} \nsubseteq \mathrm{M}_{\mathrm{R}}$. Now, suppose that $\mathrm{P}+\mathrm{Q}=\mathrm{R}$ and $\mathrm{P} \in \mathrm{M}_{\mathrm{R}}$ but $\mathrm{Q} \notin \mathrm{M}_{\mathrm{R}}$. Then there exits $T \in M_{R}$ such that $Q$ is adjacent to $T$. Since $R$ is a principal ideal ring, then $Q+(T \cap P)=$ $(\mathrm{Q}+\mathrm{T}) \cap(\mathrm{Q}+\mathrm{P})=\mathrm{T} \cap \mathrm{R}=\mathrm{T}$. Thus $\mathrm{Q}$ is adjacent to $\mathrm{T} \cap \mathrm{P}$. Since $\mathrm{P}$ is also adjacent to $\mathrm{T} \cap \mathrm{P}$, then $\mathrm{d}(\mathrm{P}, \mathrm{Q})$ $\leq 2$. Finally, diamMG(R) $\leq 2$.

The next result discovers the characterizations of the cut-vertices of MG(R).

Theorem 4.4: Suppose that every two distinct maximal ideals of $\mathrm{R}$ have a non-zero intersection. If $\mathrm{L}$ is a cut-vertex of $M G(R)$, then $L=P \cap Q$, for some $P, Q \in M_{R}$.

Proof: If $L \in M_{R}$, then by setting $M=N=L$, the proof will be completed. Now, suppose that $L \notin M_{R}$. Let $\mathrm{J}$ and $\mathrm{K}$ be two vertices in different components of $\mathrm{MG}(\mathrm{R})-\mathrm{L}$. We have three cases:

Case1: If $J, K \in M_{R}$, then $J \cap K \in N(J) \cap N(K)$. Since $L$ is a cut-vertex of $M G(R)$, then $L=J \cap K$. 
Case2: If $J \in M_{R}$ and $K \notin M_{R}$, then $K \in N(S)$, for some $S \in M_{R}$. Since $J \cap S$ is adjacent to $J$ and $S$, then $\mathrm{L}=\mathrm{J} \cap \mathrm{S}$.

Case3: If $J, K \notin M_{R}$, then $P \in N(J)$ and $Q \in N(K)$, for some $P, Q \in M_{R}$ such that $P$ and $Q$ are adjacent to $J$ and $K$, respectively. Since $I$ is a cut-vertex, then $P \neq Q$. By the same way of Case2, we obtain that $\mathrm{L}=\mathrm{P} \cap \mathrm{Q}$.

In the next main result, we find the radius of $M G(R)$.

Theorem4.6: Let $J_{R} \neq(0)$. If $\left|M_{R}\right| \geq 2$, then $\operatorname{rad}(M G(R))=2$.

Proof: From Lemma2.2, $d\left(J_{R}, K\right)=1$, for every $K \in M_{R}$. Since every vertex $I \notin M_{R}$ is adjacent to a vertex in $M_{R}$, then $d\left(J_{R}, I\right) \leq 2$. Assume that $P, Q \in M_{R}$ with $P \neq Q$. If $P Q$ is adjacent to $J_{R}$, then $\mathrm{J}_{R}+\mathrm{PQ}=\mathrm{P}$, for some $\mathrm{P} \in \mathrm{M}_{\mathrm{R}}$. Since $\mathrm{J}_{\mathrm{R}}+\mathrm{PQ} \subseteq \mathrm{P}, \mathrm{Q}$, then $\mathrm{P}=\mathrm{P}=\mathrm{Q}$. This contradicts that $\mathrm{P} \neq \mathrm{Q}$. Therefore, $P Q$ is not adjacent toJ $J_{R}$. Thus the eccentrisity of $J_{R}$ is e $\left(J_{R}\right)=2$. If there exists $I \in V(M G(R))$ with e (I) $=1$, then $I$ is adjacent to each vertex $J \in M_{R}$. Clearly, $I \notin M_{R}$. Since PQ is not adjacent toJ $J_{R}$, for every $M, Q \in M_{R}$ with $P \neq Q$, then neither $I=J_{R}$ nor $I=P Q$. Thus $I \nsubseteq J_{R}$. Hence $M G(R)$ contains a $P \in M_{R}$ which is adjacent to $\mathrm{I}$. This contradicts that $\mathrm{e}(\mathrm{I})=1$. Therefore $\mathrm{J}_{R}$ has the minimum eccentricity over all vertices of $M G(R)$. So, $\operatorname{rad}(M G(R))=e\left(J_{R}\right)=2$.

\section{References}

1. Beck, I. 1988. Coloring of Commutative ring. J. of Algebra, 116(1): 208-226.

2. Salehifar, S., Khashyarmanish, K. and Afkhami, M. 2017. On the annihilator-ideal graph of commutative rings, J. of Algebraic Com., 66(2): 431-447.

3. Gupta, R, Sen, S. M. K. and Ghosh, S. 2015. A variation of zero-divisor graphs, Discuss. Math. Gen. Algebra Appl, 35(2): 159-176.

4. $\mathrm{Yu}, \mathrm{H}$. Y. and $\mathrm{Wu}, \mathrm{T}$. 2015. Commutative rings $\mathrm{R}$ whose $\mathrm{C}(\mathrm{AG}(\mathrm{R})$ consists only of triangles. $J$. of Comm. Algebra, 43(3): 1076-1097.

5. Pirzada, S. and Raja, R. 2016. On the metric dimension of a zero-divisor graph, J. of Comm. Algebra, 45(4): 1399-1408.

6. Gary, C. and Linda, L. 1986. Graphs and Digraphs, 2nd ed., Wadsworth and Brook/ Cole , California.

7. Foldes, S. and Hammer, P.L. 1977. Split graphs, Proceedings of the 8th South-Eastern Conference on Combinatorics: Graph Theory and Computing, pp. 311-315.

8. Atiyah, M. F. and Macdonald, I. G. 1969. Introduction to Commutative Algebra. London: Addison-Wesley Publishing Co; Don Mills, Ont.

9. Berge, C. 1961. Farbung von Graphen deren sämtliche beziehungsweise deren ungerade Kreise Starr Sind, Wissenschaftliche Zeitschrift, Martin Luther Univ. Halle-Wittenberg, Math.-Naturwiss. Reihe, 114-115,

10. David, S. and Richard, M. 1991. Abstract Algebra. U. S. A.: Prentice-Hall Inc. 